\title{
Rehabilitative treatment of cleft lip and palate: experience of the Hospital for Rehabilitation of Craniofacial Anomalies/USP (HRAC/USP) - Part 5: Institutional outcomes assessment and the role of the Laboratory of Physiology
}

\author{
José Alberto de Souza FREITAS ${ }^{1}$, Ivy Kiemle TRINDADE-SUEDAM², Daniela Gamba GARIB ${ }^{3}$, Lucimara Teixeira \\ das NEVES ${ }^{4}$, Ana Lúcia Pompéia Fraga de ALMEIDA ${ }^{5}$, Renato Yassukata Faria YAEDÚ ${ }^{6}$, Thaís Marchini OLIVEIRA ${ }^{7}$, \\ Simone SOARES ${ }^{5}$, Rita de Cássia Moura Carvalho LAURIS ${ }^{8}$, Renata Paciello YAMASHITA ${ }^{9}$, Alceu Sergio TRINDADE \\ $\mathrm{JR}^{10}$, Inge Elly Kiemle TRINDADE ${ }^{11}$, João Henrique Nogueira PINTO ${ }^{12}$
}

\begin{abstract}
1- DDS, MSc, PhD, Superintendent, Hospital for Rehabilitation of Craniofacial Anomalies and Full Professor, Department of Stomatology, Bauru School of Dentistry, University of São Paulo, Bauru, SP, Brazil.

2- DDS, MSc, PhD, Assistant Professor, Department of Biological Sciences, Bauru School of Dentistry and Laboratory of Physiology, Hospital for Rehabilitation of Craniofacial Anomalies, University of São Paulo, Bauru, SP, Brazil.

3- DDS, MSc, PhD, Associate Professor, Department of Pediatric Dentistry, Orthodontics and Community Health, Bauru School of Dentistry and Hospital for Rehabilitation of Craniofacial Anomalies, University of São Paulo, Bauru, SP, Brazil.

4- DDS, MSc, PhD, Assistant Professor, Department of Biological Sciences, Bauru School of Dentistry and Hospital for Rehabilitation of Craniofacial Anomalies, University of São Paulo, Bauru, SP, Brazil.

5- DDS, MSc, PhD, Assistant Professor, Department of Prosthodontics, Bauru School of Dentistry and Hospital for Rehabilitation of Craniofacial Anomalies, University of São Paulo, Bauru, SP, Brazil.

6- DDS, MSc, PhD, Assistant Professor, Department of Stomatology, Bauru School of Dentistry and Hospital for Rehabilitation of Craniofacial Anomalies, University of São Paulo, Bauru, SP, Brazil.

7- DDS, MSc, PhD, Assistant Professor, Department of Pediatric Dentistry, Orthodontics and Community Health, Bauru School of Dentistry and Hospital for Rehabilitation of Craniofacial Anomalies, University of São Paulo, Bauru, SP, Brazil.

8- DDS, MSc, Orthodontist of the Dental Division of the Hospital for Rehabilitation of Craniofacial Anomalies, University of São Paulo, Bauru, SP, Brazil. 9- BA, MSc, PhD, Speech Pathologist, Laboratory of Physiology, Hospital for Rehabilitation of Craniofacial Anomalies, University of São Paulo, Bauru, SP, Brazil. 10- DDS, MSc, PhD, Full Professor, Department of Biological Sciences, Bauru School of Dentistry and Laboratory of Physiology, Hospital for Rehabilitation of Craniofacial Anomalies, University of São Paulo, Bauru, SP, Brazil.

11- BS, MSc, PhD, Full Professor, Department of Biological Sciences, Bauru School of Dentistry and Laboratory of Physiology, Hospital for Rehabilitation of Craniofacial Anomalies, University of São Paulo, Bauru, SP, Brazil.

12- DDS, MSc, PhD, Prosthodontist of the Dental Division of the Hospital for Rehabilitation of Craniofacial Anomalies, University of São Paulo, and University of Sacred Heart (USC), Bauru, SP, Brazil.
\end{abstract}

Corresponding address: Ivy Kiemle Trindade-Suedam - Hospital for Rehabilitation of Craniofacial Anomalies, University of São Paulo - Rua Dr. Silvio Marchione 3-20, 17012-900, Bauru-SP, Brazil - Phone: +55(14) 3235-8282 - Fax: +55(14) 3223-4679 - e-mail: ivysuedam@fob.usp.br

Received: April 12, 2013 - Accepted: May 2, 2013

\section{ABSTRACT}

The Laboratory of Physiology provides support for the diagnosis of functional disorders
associated with cleft lip and palate and also conducts studies to assess, objectively, the
institutional outcomes, as recommended by the World Health Organization. The Laboratory
is conceptually divided into three units, namely the Unit for Upper Airway Studies, Unit for
Stomatognathic System Studies and the Unit for Sleep Studies, which aims at analyzing the
impact of different surgical and dental procedures on the upper airways, stomatognathic
system and the quality of sleep of individuals with cleft lip and palate. This paper describes
the main goals of the Laboratory in the assessment of procedures which constitute the
basis of the rehabilitation of cleft lip and palate, i.e., Plastic Surgery, Orthodontics and
Maxillofacial Surgery and Speech Pathology.

Key words: Cleft palate. Physiology. Outcome and process assessment. 


\section{INTRODUCTION}

The Hospital for Rehabilitation of Craniofacial Anomalies (HRAC) of the University of São Paulo (USP), acknowledged by the World Health Organization as a world reference center for the treatment of craniofacial anomalies, including cleft lip and palate, is distinguished by its expressive work in teaching, research and extension activities. Official data of the yearly statistics of USP from $2011^{27}$ indicate that more than 7,000 surgeries were performed at HRAC in that year, accounting for approximately 660 surgeries/month. To achieve those numbers, the HRAC has an interdisciplinary team characterized by humanistic care and a strong scientific nature.

With the establishment of the Post Graduate Program in Rehabilitation Sciences in the 1990s and the increasing need to adopt therapeutic approaches based on scientific evidences, the assessment of institutional outcomes became a common goal in the philosophy of team professionals, who aim to establish the best protocols by their researches, offering a high quality treatment for individuals with cleft lip and palate. This philosophy meets the guidelines suggested by Shaw and Semb ${ }^{15}$ (2007), researchers of the University of Manchester-UK and scientific mentors of HRAC-USP, who state that professionals involved in the rehabilitation of cleft lip and palate should be committed with the assessment of the efficiency of their outcomes. Long $^{12}$ (1996) defined this practice as "a systematic critical analysis of the quality of care offered, including diagnostic and therapeutic procedures, resources employed, outcomes achieved and the quality of life offered to individuals".

Within this context, in 2002, a group of professionals from the world cleft centers, including HRAC, met in Geneva, Switzerland, under the support of the World Health Organization (WHO) to discuss the world strategies to reduce the damages in the treatment offered to individuals with craniofacial anomalies ${ }^{33}$. Among the several recommendations, this task force emphasized the need of prospective and controlled clinical studies to assess the outcomes of treatment adopted by each center and later, an inter-center comparison, for definition of the best protocols and therapeutic approaches to be adopted internationally.

The HRAC Laboratory of Physiology is included in this context, as well as other laboratories and divisions of the institution, which provide support to the diagnosis of functional disorders associated to cleft lip and palate and also conduct studies to assess the institutional outcomes. In particular, the Laboratory of Physiology is conceptually divided into three units, namely the Unit for Upper Airway Studies, Unit for Stomatognathic System Studies and the Unit for Sleep Studies, whose investigations aim at analyzing the impact of different surgical and dental procedures on the upper airways, stomatognathic system and the quality of sleep of individuals with cleft lip and palate.

Thus, this paper describes the role of the HRAC Laboratory of Physiology in the assessment of institutional outcomes in the fields, which constitute the basis of rehabilitation of the cleft lip and palate: Medicine, particularly plastic surgery; Dentistry, particularly, Orthodontics and Maxillofacial Surgery; and Speech Pathology.

\section{THE LABORATORY OF PHYSIOLOGY AND ITS RELATIONSHIP WITH PLASTIC SURGERY AND SPEECH PATHOLOGY}

Plastic surgery for lip repair is ideally performed around three months of age and represents the first among many surgical interventions to which the individual with cleft lip and palate is submitted for full rehabilitation. This surgical procedure reconstructs the skin, muscle, lip and oral mucosa, as well as the nasal columella. Even though this procedure immediately restores the continuity of facial structures disrupted by the cleft, with a shortterm positive effect on the orofacial functions and especially on the facial esthetics, this procedure ultimately creates a fibrous tissue that restricts the full maxillary skeletal growth and development over time, leading to Angle Class III malocclusion in a considerable proportion of individuals. In a study conducted at the HRAC Laboratory of Physiology, Genaro, Trindade Jr and Trindade ${ }^{6}$ (1994) demonstrated by electromyography that the orbicularis oris muscle of young individuals with unilateral cleft lip and palate presents significantly greater action potentials than individuals without cleft, both at rest and during speech, suggesting that this increased electromyographic activity may contribute to the deficit of maxillary growth commonly observed in this population.

The clefts may affect the lip and palate simultaneously. Palatoplasty is performed around the age of 12 months. Nasal shape and function are also affected by clefts which involve the lip and palate. In unilateral clefts, the two portions of the orbicularis oris muscle separated by the cleft tend to pull the nasal structures in opposite directions; the force is applied on the external alar base at the cleft area and on the columella base at the opposite side. Consequently, the nasal pyramid is deviated to the non-cleft side and the nostril is flattened on the cleft side. Conversely, in symmetric bilateral clefts, the nose is not deviated and the most evident alterations are the short or even absent columella with a flattened and widened nasal tip; in asymmetrical cases, which are less frequent, the 
imbalance of forces produce deformities similar to those of unilateral clefts. These deformities often reduce the internal nasal dimensions, increasing the nasal resistance to the airflow and leading to mouth breathing, which affects the craniofacial development ${ }^{1}$.

The correction of these nasal deformities, common in individuals with clefts, involves surgical correction of the deviated nasal septum, nasal bone structures and also of the tip, base and ala of the nose. Rhinoseptoplasty and columela lengthening are usually performed around six years of age. Even though there is consensus on the positive effects of rhinoseptoplasty on nasal esthetics, the impact of this surgery on the nasal function, by that time not fully understood, was investigated in 2009 by Trindade, Beriter and Sampaio-Teixeira ${ }^{20}$. The authors objectively assessed the internal nasal dimensions and speech nasality in individuals with complete unilateral cleft lip and palate submitted to rhinoseptoplasty, by acoustic rhinometry and nasometry, respectively. In most cases there was a persistent increase of the internal nasal dimensions and consequently of the nasal patency, yet without changes in speech resonance. These data have shown the success of the procedure concerning the functional aspects with a significant improvement in breathing without a negative effect on speech, which is already impaired in individuals with cleft lip and palate.

Cleft palate speech is primarily characterized by hypernasality caused by the anatomical impairment of the velopharyngeal structures, which act like a sphincter, responsible for the separation of the nasal and oral cavities during production of speech oral sounds or during swallowing. The failure in sphincter closure, generically called velopharyngeal dysfunction (VPD), is caused by lack of tissue, i.e. a short soft palate (velopharyngeal insufficiency), or by a deficiency of palatal mobility (velopharyngeal incompetence). This leads to typical speech alterations which may persist even after primary correction of the palatal defect. These are basically three characteristics: 1) nasal air escape during speech, audible or not, 2) weak intraoral pressure, and consequently 3 ) excessive nasal resonance clinically known as hypernasality. To avoid these disorders, compensatory articulations may happen, such as the production of the plosive /p/ at the glottal level, the so-called glottal stop, which conversely may impair speech intelligibility.

The Physiology team has been using aerodynamic and acoustic methods to objectively evaluate the results of "speech surgeries", aiming to correct velopharyngeal insufficiency by creating a tissue bridge between the posterior pharyngeal wall and the soft palate (pharyngeal flap surgery), thus establishing a mechanical obstruction separating the nasal cavity from the remaining vocal tract or by surgical repositioning of the soft palate musculature (intravelar veloplasty), elongating it, consequently enhancing its mobility and ultimately promoting adequate closure. The pharyngeal flap surgery has been the most used technique to correct velopharyngeal insufficiency in different reference centers around the world. However, intravelar veloplasty, among other techniques, has been increasingly used because it has been considered a "more physiological" procedure ${ }^{18}$.

The perceptual speech evaluation is the main indicator of the clinical significance of symptoms caused by VPD and, as such, it is fundamental for the diagnosis, along with physical examination and clinical history survey ${ }^{11}$. Based on the perceptual assessment of resonance (nasal or oral) and the presence or absence of nasal air emission and compensatory articulations during speech, it is possible to categorize the velopharyngeal function. However, despite its known importance, the perceptual evaluation presents some limitations related to its subjectivity. Thus, it became necessary to use objective methods to complement the clinical impressions ${ }^{23}$.

The instrumental methods currently available may be grouped into two categories, namely direct and indirect. The direct methods allow visualization of the velopharyngeal anatomy, i.e. of the structures involved in velopharyngeal closure, and how they move during different activities. This is the case of nasoendoscopy and videofluoroscopy. Conversely, the indirect methods provide information related to the physiology of velopharyngeal activity, i.e. on the aerodynamic and acoustic repercussions of velopharyngeal functioning. This is the case of rhinomanometry and nasometry.

Rhinomanometry, also called the flow-pressure technique, provides quantitative information on the dimensions of the velopharyngeal orifice during speech. It is based on the principle that the area of a constriction (or orifice) may be calculated by knowing the differential pressure between the two sides of the constriction and the airflow through it. Examining is usually performed with a computer system called the PERCI (abbreviation for Palatal Efficiency Rating Computed Instantaneously). The technique allows calculation of the minimum cross-sectional area of the velopharynx, i.e. of the velopharyngeal orifice, during the production of an oral sound. The differential pressure is measured through the orifice by positioning a catheter inside the mouth and another in one nostril. The nasal catheter is kept in place with the aid of an obturator that blocks the nostril, thus creating a static air column. Both catheters are connected to pressure transducers. Nasal airflow is measured on the other nostril by using a heated pneumotachograph, 
whose proximal end is connected to the nostril with a plastic tube and the distal end is connected to a pressure transducer. The signals of the three transducers are sent to the PERCI system for analysis by a specific software.

The measurement is performed during production of the voiceless plosive consonant /p/, usually inserted in the word hamper in English and in the word "rampa" in Portuguese, as done at the HRAC Laboratory of Physiology, because this sound requires the maximum movement of the soft palate. Four to six productions are recorded and, based on the measurements of differential pressure and flow, it is possible to calculate the resistance offered by the velopharyngeal orifice, or its cross-sectional area. According to the area obtained, the velopharyngeal closure may be classified as adequate when smaller than $5 \mathrm{~mm}^{2}$, marginal between 5 and $19 \mathrm{~mm}^{2}$ and inadequate when greater than $20 \mathrm{~mm}^{2}$. Knowledge on these values allows analysis of the adequacy of the velopharyngeal mechanism in normal and altered conditions. Trindade, et al. ${ }^{24}$ (2003), for example, used the technique to assess the effects of maxillary advancement on velopharyngeal closure in the short and long term. Miguel, Genaro and Trindade $^{14}$ (2007) characterized velopharyngeal closure in individuals with submucous cleft palate and Fukushiro and Trindade ${ }^{5}$ (2011) in patients with pharyngeal flap.

Nasometry, in turn, estimates speech resonance by measuring nasalance, which corresponds to the relative quantity of acoustic energy emerging from the nasal cavity during speech. Nasalance is the acoustic correlate of nasality, which corresponds to the subjective perception of a listener on the nasal component of speech. Evidences demonstrate good correlation between measurements of nasalance and the perceptual judgment of nasality ${ }^{30}$.

The nasometer is composed of two microphones positioned one at each side of a sound separation plate, which is positioned against the upper lip; the assembly is kept in place with a headgear. The upper microphone captures signals of the nasal component of speech, and the lower microphone captures signals of the oral component. As the individual reads standard speech samples presented on the computer screen, the acoustic signals are filtered and digitized and then analyzed by a specific software. Nasalance is calculated by the numeric ratio between the nasal acoustic energy and the total acoustic energy (sum of nasal and oral acoustic energies), multiplied by 100 , i.e.: $\%$ nasalance $=$ nasal/oral+nasal $\times 100$. Thus, nasalance is expressed in percentage and may theoretically vary between $0 \%$ (absence of nasal sound) and $100 \%$ (all sound emerging from the nose). The assessment of the velopharyngeal function is conventionally performed during the reading of standardized sentences without nasal sounds. In this case, an increased nasalance score indicates hypernasality and indirectly the presence of VPD. The nasalance score thus obtained may be compared with reference values determined for normal subjects, as those from Trindade and Dalston $^{22}$ (1997). They may be also compared to the normal limits, which is $27 \%$ for the sentences containing exclusively oral sounds. Values higher than $27 \%$ are suggestive of hypernasality. The normal limit is $43 \%$ for sentences containing nasal sounds, i.e. values lower than $43 \%$ suggest hyponasality.

Within this context, the effectiveness of pharyngeal flap surgery was analyzed in 240 individuals with cleft lip and palate and velopharyngeal insufficiency, measuring the speech nasalance by nasometry and the velopharyngeal closure by rhinomanometry, immediately before and one year after surgery ${ }^{5}$. In a considerable number of cases, there was improvement of velopharyngeal closure with a significant reduction of the nasalance scores, especially in children finalizing speech therapy.

The effect of secondary palatoplasty with intravelar veloplasty on the nasalance of 40 individuals with repaired cleft palate and velopharyngeal insufficiency was assessed immediately before and 8 months after surgery by Yamashita, et al. ${ }^{28}$ (2012). The authors compared the outcomes according to the degree of velopharyngeal closure before surgery (small gaps or medium and large gaps), as analyzed by a nasopharyngoscopy. The authors concluded that intravelar veloplasty improved the speech resonance in most individuals analyzed and that surgery was more effective in the correction or reduction of nasalance of individuals presenting small gaps before surgery. Thus, as demonstrated for pharyngeal flap surgery ${ }^{5}$, the intravelar veloplasty was shown to be effective in reducing the nasalance during speech.

Conversely, the findings of Yamashita and Trindade $^{29}$ (2008) had demonstrated that the pharyngeal flap may also lead to obstructive respiratory symptoms during sleep. A systemic review conducted by the team of the Laboratory of Physiology confirmed the significant potential for upper airway obstruction caused by the pharyngeal flap ${ }^{3}$. Reports of the literature have shown that respiratory disorders associated to the presence of the flap include hyponasality, snoring, nasal obstruction and, more importantly, obstructive sleep apnea syndrome (OSAS). OSAS is characterized by recurrent events of upper airway obstruction during sleep, which are manifested as reduction (hypopnea) or interruption (apnea) of the respiratory airflow in the presence of thoracic effort, frequently leading to microarousals. Polysomnography, considered 
the gold standard method for the diagnosis of obstructive sleep apnea ${ }^{8}$, consists of simultaneous recording of different physiological variables during sleep, which include the oronasal airflow, arterial oxygen desaturation and others assessed by electroencephalography, electrooculography, electromyography and electrocardiography.

Therefore, the HRAC Laboratory of Physiology has been focusing on the investigation of sleep aspects of individuals with cleft and the obstructive respiratory events caused by speech surgeries to establish treatment protocols based on scientific evidences. A Unit for Sleep Studies was set up for that purpose, with a EMBLA N7000 polysomnography system. Considering that the increase in age is a known factor influencing the prevalence of OSAS, affecting 38 to $49 \%$ of the population without cleft ${ }^{26}$, an ongoing study aims to prospectively analyze the occurrence and severity of OSAS in middle-aged individuals with cleft lip and palate and pharyngeal flap as compared to a group of individuals with cleft lip and palate without pharyngeal flap. Preliminary results in individuals who underwent full-night polysomnography, 20 years after surgery, on average, suggest that, regardless of the presence of the flap, the older patients with cleft present a higher prevalence of OSAS than the general population ${ }^{26}$. The authors assign these findings to anatomical and functional factors inherent to the cleft, besides advancing age, which should be further investigated.

\section{THE LABORATORY OF PHYSIOLOGY AND DENTISTRY}

Dentistry, like Plastic Surgery and Speech Pathology, constitutes one of the most representative specialties among many working with cleft lip and palate care. Among the several dental specialties, Orthodontics and Oral and Maxillofacial Surgery, in particular, represent part of the treatment that is anxiously expected by patients, because they correct deficiencies that compromise the facial esthetics, including dental malocclusion and skeletal facial alterations, respectively.

In general, the dentistry protocol of the HRAC begins with diagnosis and pediatric dental care. Preventive orthodontic treatment starts in the early mixed dentition to enhance the interarch relationship and correct transverse and anteroposterior maxillary discrepancies by rapid maxillary expansion (RME) and maxillary protraction, respectively. Maxillary atresia should be ideally corrected before alveolar bone graft surgery $(A B G)$, whose main objective is to provide bone tissue at the cleft area and consequently restore the continuity of the alveolar ridge, stabilizing the maxilla and providing bone support to the nasal alar base, improving the nasal symmetry ${ }^{9}$.

Considering the importance of RME and ABG for the rehabilitation of individuals with cleft lip and palate, researchers of the Laboratory of Physiology developed studies to investigate the impact of these procedures on the upper airway of these individuals. Trindade, et al. ${ }^{21}$ (2010) prospectively assessed the effect of RME on the nasal patency of individuals with unilateral cleft, by acoustic rhinometry. An ongoing study is investigating the impact of RME in individuals with bilateral clefts. Acoustic rhinometry is based on the physical principle that the sound, traveling through the nasal cavity, is reflected back by constrictions in its way up to the nasopharynx. The technique comprises the analysis of sounds reflected by the nasal cavity in response to incident waves emitted by a sound source positioned at the distal end of the tube called the rhinometer, which is positioned against one nostril7. The sound pressure signals are captured by a microphone, which is also positioned inside the tube, and analyzed by a software that allows determination of sectional areas of the entire nasal cavity, visualized on an area-distance curve - the rhinogram. The volumes of different segments of the nose and nasopharynx are calculated from the integration of the areaunder the curve ${ }^{1}$.

In the study of Trindade, et al. ${ }^{21}$ (2010), individuals with unilateral cleft were submitted to acoustic rhinometry before placement of the expander and after the active stage of expansion. The study assessed the sectional areas and volumes of the nasal segments corresponding to the valve region (AST1 and $\mathrm{V} 1$ ) and nasal turbinates (AST2, AST3 and V2) after nasal decongestion. The results showed that, in the group as a whole, the RME promoted a statistically significant increase in AST1 and V1. The individual analysis of the data demonstrated that most cases responded positively to RME, with a mean increase of $26 \%$. In addition, an ongoing study showed that individuals analyzed with bilateral clefts had significant increases in all variables and means, except for AST3, which corresponds to the sectional area of the posterior turbinate region. Individual data showed that $74 \%$ of cases responded positively to RME, with a mean increase of $40 \%$, demonstrating that the group of individuals with bilateral cleft was much more susceptible to the effect of RME. This may be due to the greater expansion usually required by these cases, because of the greater maxillary atresia observed in individuals with bilateral cleft compared to those with unilateral cleft. Overall, these findings demonstrated that RME increases the internal nasal dimensions, consequently leading to an increase of nasal permeability to the airflow, thereby favoring the nasal breathing.

As previously mentioned, the correction of 
maxillary atresia is followed by alveolar bone grafting $(A B G)$. This procedure has the main objective of providing bone tissue for the remaining alveolar cleft and, secondarily, to reconstruct the nose at the cleft area. Because nasal cavities are impaired in individuals with clefts affecting the lip and the alveolar process, it became relevant to investigate the actual impact of the ABG procedure on the nasal geometry. Preliminary results from the Physiology team at the HRAC have shown that subjects with complete unilateral cleft lip and palate underwent acoustic rhinometry before and 60-120 days after ABG surgery. An increase of nasal sectional areas and volumes was observed after surgery in $50 \%$ of the sample. However, in the remaining $40 \%$ cases, a reduction was seen. These conflicting data raise considerations on the two possible effects of ABG surgery on the nasal cavity, namely: in some cases, the ABG surgery may increase the nasal patency, possibly by the supporting effect of the graft on the alar base of the nose, opening the previously collapsed nasal valve; in other cases, the ABG reduces the nasal patency due to elevation of the nasal floor inside the nasal cavity, which was previously invaginated in the cleft area, thus reducing its internal dimensions. Further studies are being conducted to elucidate those issues.

The HRAC new protocol comprises the onset of corrective orthodontic treatment after ABG surgery, aiming at tooth alignment and leveling with or without orthognathic surgery, especially in cases diagnosed with the occlusal scores Goslon 4 and $5^{13}$. The referral to orthognathic surgery in skeletal maturity occurs when the maxillomandibular discrepancy surpassed the possibility of action of orthodontic and orthopedic resources. The surgery aims to correct the occlusal disharmonies, especially Class III, providing a balanced interocclusal relationship. With reestablishment of the interarch relationship, the occasional prosthetic planning is then possible, aiming at the esthetic and occlusal outcomes ${ }^{16}$.

Even though several clinical studies have demonstrated high success rates of orthognathic surgery for the correction of maxillomandibular discrepancies in individuals with cleft lip and palate, the functional effects of the procedure on speech and breathing has not yet been fully elucidated. Clinical observations have suggested that reestablishment of the occlusal balance and consequent adjustment of lip and teeth position enhance speech articulation ${ }^{4}$. Also, it was anticipated that the orthognathic surgery benefits breathing, because procedures such as maxillary advancement might increase nasopharyngeal space and widen the nasal valve, favoring nasal patency ${ }^{31,32}$. However, at that time, it was questioned whether the maxillary advancement might lead to hypernasality or even contribute to worsen a preexisting hypernasality, because of the effect on velopharyngeal closure.

Therefore, Trindade, et al. ${ }^{24}$ (2003) used rhinomanometry and nasometry to evaluate the short- and long-term effects of maxillary advancement on the aerodynamic and acoustic aspects of velopharyngeal function. In the short term, the authors observed an increase of the velopharyngeal orifice area (gap) with the consequent increase of nasalance. However, in the long term, the orifice area returned to its original dimensions, which was assigned to the accommodation of pharyngeal structures to the new dimensions of the gap. Yet the nasalance remained increased. The authors assigned this finding to the dilating effect of maxillary advancement on the nasal valve, reducing nasal resistance, and paradoxically favoring nasal air escape and nasal resonance.

Considering that a cleft dramatically alters the dentomaxillary anatomy and that the homeostasis of the stomatognathic system, especially of its musculature, is associated with occlusal balance, the Laboratory of Physiology also evaluated the muscular activity of individuals with cleft lip and palate by assessing the duration of the electromyographic silent period (ESP) of the mandibular levator muscles ${ }^{25}$. The ESP corresponds to the time during which the action potential of muscles are not recorded, even during their contraction, due to temporary inhibition of the trigeminal motor nucleus by stimulation of muscle spindles and periodontal mechanoreceptors. Classical studies ${ }^{2,19}$ demonstrated that this kind of measurement is used as diagnostic tool because the ESP duration is systematically increased in electromyographic records of individuals with dysfunctions of the stomatognathic system. Thus, individuals with unilateral cleft lip and palate were compared to individuals with isolated cleft lip and individuals without cleft. ESP observed in the group with cleft involving lip and palate was significantly longer, demonstrating that the masticatory system is impaired in this population and that the measurement of ESP duration is a good method for assessing the impact of cleft lip and palate on the functional status of the stomatognathic system ${ }^{25}$.

Another indicator of the masticatory performance is the analysis of the bite force. A study conducted at the Laboratory of Physiology by Sipert, et al. ${ }^{17}$ (2009) quantified the bite force in control individuals (without cleft lip and palate and other malocclusions and free of signs and symptoms of temporomandibular dysfunction) and a group of individuals with unilateral cleft lip and palate (undergoing corrective orthodontic treatment and free of signs and symptoms of temporomandibular 
dysfunction). Lower bite force values were observed for the group with cleft, yet the differences were statistically significant only for the male gender. Because no differences were found between the cleft and non-cleft maxillary sides, authors concluded that the reduced bite force in individuals with cleft lip and palate seem to be more related to the orthodontic treatment than to the cleft itself. Ongoing studies are being conducted to further analyze these aspects.

\section{INTERNATIONALIZATION}

The Laboratory of Physiology has been increasingly targeted to internationalization, participating with multicenter studies with the main goal of establishing protocols based on scientific evidences.

The project "Timing of Primary Surgery for Cleft Palate", known by its acronym TOPS, is an international multicenter study conducted in 23 centers of the United Kingdom, Scandinavia and Brazil, in this case at the Hospital for Rehabilitation of Craniofacial Anomalies of USP, Bauru-SP. The project is supported by the National Institutes of Health/National Institute of Dental and Craniofacial Research (NIH/NIDCR) of the United States and the leader in Brazil is a professor and investigator of the Laboratory of Physiology. The main objective of this trial is to determine the best age ( 6 or 12 months) for primary surgery in infants with isolated cleft palate, to achieve the best outcomes as to speech, hearing and craniofacial growth.

The Exchange Project CAPES/FIPSE involves partnership with two Brazilian institutions, the Federal University of Santa Maria and the University of São Paulo, including the Hospital for Rehabilitation of Craniofacial Anomalies and Bauru School of Dentistry; and two North American institutions, the East Tennessee State University and University of Northern Iowa. It is part of the US-Brazil Higher Education Consortia Program, a collaboration between Capes (Coordination for the Improvement of Higher Education Personnel) and FIPSE (Fund for the Improvement of Postsecondary Education) of the United States. The main objective of this project is to promote the exchange of students and collaboration in studies for "the understanding of differences and similarities of communication disorders in Brazilian Portuguese and English speaking children", currently under way and also coordinated at USP by a professor and investigator of the Laboratory of Physiology.

Finally, it should be highlighted that researchers of the Laboratory of Physiology have also established important national partnerships, involving other units of USP and other institutions, conducting advanced studies on the physiology of the upper airway and stomatognathic system. In combination, these partnerships have significantly contributed to advances in knowledge and to the differentiated training of undergraduate and postgraduate students from Brazil and abroad.

\section{FINAL CONSIDERATIONS}

The assessment of institutional outcomes by objective analysis methods is one of the main roles of the HRAC Laboratory of Physiology. The continuous involvement with research, its original vocation, gives support to diagnosis and guides clinical work. One of the examples of a clinical routine adjusted according to the outcomes of studies developed at the Laboratory, is that, at present, all patients referred by the clinical team for surgical correction of velopharyngeal dysfunction, are necessarily assessed at the Laboratory of Physiology preoperatively and also postoperatively for the evaluation of surgical outcomes.

Finally, the importance and influence of Prof. Dr. Miguel Rolando Covian, Prof. Dr Eduardo Moacyr Krieger, Prof. Dr. José Carlos Manço and Prof. Dr. Donald Warren should be highlighted, as they have guided, as true scientific mentors, the whole Physiology team at HRAC. And also Professors Dr. Alceu Trindade and Dra. Inge Trindade, who set up the Laboratory and trained more than 45 masters and doctors so far. As highlighted by Krieger ${ }^{10}$ (2004), they have put into practice the actual meaning of "Integrated Knowledge" and "Translational Physiology", speeding the transference of research outcomes, diagnosis and treatment of cleft lip and palate.

\section{ACKNOWLEDGMENTS}

We are thankful to FAPESP, CAPES and CNPq for the financial support and to Assistant Professor Ana Paula Fukushiro, Associate Professor Kátia Flores Genaro and Dr. Ana Cláudia Martins SampaioTeixeira by joint collaboration over the years.

\section{REFERENCES}

1- Bertier CE, Trindade IEK. Deformidades nasais: avaliação e tratamento cirúrgico. In: Trindade IEK, Silva Filho OG, editors. Fissuras labiopalatinas: uma abordagem interdisciplinar. São Paulo: Ed. Santos; 2007. p. 87-107.

2- Bessette R, Bishop B, Mohl N. Duration of masseteric silent period in patients with TMJ syndrome. J Appl Physiol. 1971;30:864-9.

3- Cardia CCO, Yamashita RP, Campos LD, Sampaio-Teixeira ACM, Trindade-Suedam IK, Trindade IEK. Obstrução respiratória após cirurgia de retalho faríngeo para correção de insuficiência velofaríngea revisão da literatura. Rev Soc Bras Cir Craniomaxilofac. 2011;14:207-13.

4- Dalston RM, Vig PS. Effects of orthognathic surgery on speech: a prospective study. Am J Orthod. 1984;86:291-8. 
5- Fukushiro AP, Trindade IE. Nasometric and aerodynamic outcome analysis of pharyngeal flap surgery for the management of velopharyngeal insufficiency. J Craniofac Surg. 2011;22:164751.

6- Genaro KF, Trindade Júnior AS, Trindade IE. Electromyographic analysis of lip muscle function in operated cleft subjects. Cleft Palate Craniofac J. 1994;31:56-60.

7- Hilberg O. Objective measurement of nasal airway dimensions using acoustic rhinometry: methodological and clinical aspects. Allergy. 2002;57(Suppl 70):5-39.

8- Iber C, Redline S, Kaplan Gilpin AM, Quan SF, Zhang L, Gottlieb $D$, et al. Polysomnography performed in the unattended home versus the attended laboratory setting - Sleep Heart Health Study methodology. Sleep. 2004;27:536-40

9- Kokkinos PP, Ledoux W, Kinnebrew MC, Waeinberg R. Iliac apophyseal cartilage augmentation of the deficient piriform rim and maxilla in cleft grafting. Am J Orthod Dentofacial Orthop. 1997; 112:145-53.

10- Krieger EM. Saber integrado no hospital universitário [online]. Folha de São Paulo, São Paulo, 18 Jul. 2004. [cited 12 Apr. 2013]. Available from http://www1.folha.uol.com.br/fsp/opiniao/ fz1807200408.htm.

11- Kummer AW, Briggs M, Lee L. The relationship between the characteristics of speech and velopharyngeal gap size. Cleft Palate Craniofac J. 2003;40(6):590-6.

12- Long AF. Outcomes within audit: a process and outcome perspective. Outcomes Briefing. 1996;7:4-7.

13- Mars M, Plint DA, Houston WJ, Bergland O, Semb G. The Goslon Yardstick: a new system of assessing dental arch relationships in children with unilateral clefts of the lip and palate. Cleft Palate J. 1987;24:314-22.

14- Miguel HC, Genaro KF, Trindade IE. Perceptual and instrumental assessment of velopharyngeal function in asymptomatic submucous cleft palate. ProFono. 2007;19:105-12.

15- Shaw W, Semb G. Princípios e estratégias da reabilitação: recomendações da OMS. In: Trindade IEK, Silva FIlho OG, editors. Fissuras labiopalatinas: uma abordagem interdisciplinar. São Paulo: Ed. Santos; 2007. p.1-5.

16- Silva OG Filho, Freitas JAS, Ozawa TO, Trindade-Suedam IK. Fissuras labiopalatinas: fundamentos terapêuticos. In: Melega JM, editor. Cirurgia plástica - os princípios e a atualidade. Rio de Janeiro: Guanabara Koogan; 2011. p. 285-304.

17- Sipert CR, Sampaio AC, Trindade IE, Trindade AS Jr. Bite force evaluation in subjects with cleft lip and palate. J Appl Oral Sci. 2009;17:136-9.

18- Sloan GM. Posterior pharyngeal flap and sphincter pharyngoplasty: the state of the art. Cleft Palate Craniofac J. 2000;37:112-22.

19- Smith DM, Ziter WD, Mohl ND, McCall WD Jr. Influence of auriculotemporal nerve anaesthesia on the masseteric silent period. J Oral Rehabil. 1983;10:251-6.
20- Trindade IE, Bertier CE, Sampaio-Teixeira ACM. Objective assessment of internal nasal dimensions and speech resonance in individuals with repaired unilateral cleft lip and palate after rhinoseptoplasty. J Craniofac Surg. 2009;20:308-14.

21- Trindade IEK, Castilho RL, Sampaio-Teixeira ACM, TrindadeSuedam IK, Silva Filho OG. Effects of orthopedic rapid maxillary expansion on internal nasal dimensions in children with cleft lip and palate assessed by acoustic rhinometry. J Craniofac Surg. 2010;21:306-11.

22- Trindade IEK, Dalston RM. Nasalance scores of normal Brazilian Portuguese speakers. Braz J Dysmorphol Speech Hear Disord. 1997; 1:23-34

23- Trindade IE, Genaro KF, Yamashita RP, Miguel HC, Fukushiro AP. Proposal for velopharyngeal function rating in a speech perceptual assessment. ProFono. 2005;17:259-62.

24- Trindade IE, Yamashita RP, Suguimoto RM, Mazzottini R, Trindade AS Jr. Effects of orthognathic surgery on speech and breathing of subjects with cleft lip and palate: acoustic and aerodynamic assessment. Cleft Palate Craniofac J. 2003;40:54-64. 25- Trindade Júnior AS, Westphalen FH, Trindade IE, Maringoni $\mathrm{RL}$, Atta AG. Masseteric electromyographyc silent period duration in patients with temporomandibular joint dysfunction. Braz J Med Biol Res. 1991;24:261-6.

26- Tufik S, Santos-Silva R, Taddei JA, Bittencourt LR. Obstructive sleep apnea syndrome in the São Paulo epidemiologic sleep study. Sleep Med. 2010;11:441-6

27- Universidade de São Paulo. Anuário estatístico da USP. São Paulo: USP; 2012. Available from: https://uspdigital.usp.br/ anuario/.

28- Yamashita RP, Carvalho ELL, Fukushiro AP, Zorzetto NL, Trindade IEK. Efeito da veloplastia intravelar sobre a nasalidade em indivíduos com insuficiência velofaríngea. Rev CEFAC. 2012;14:1-7.

29- Yamashita RP, Trindade IE. Long-term effects of pharyngeal flaps on the upper airways of subjects with velopharyngeal insufficiency. Cleft Palate Craniofac J. 2008;45:364-70.

30- Watterson T, Lewis KE, Deutsch C. Nasalance and nasality in low pressure and high pressure speech. Cleft Palate Craniofac J. 1998;35:293-8

31- Wetmore RF. Importance of maintaining normal nasal function in the cleft palate patient. Cleft Palate Craniofac J. 1992;29:498506.

32- Wolford LM. Effects of orthognathic surgery on nasal form and function in the cleft patient. Cleft Palate Craniofac J. 1992; 29:546-55.

33- World Health Organization. Global strategies to reduce the health-care burden of craniofacial anomalies. Report of WHO meetings on International Collaborative Research on Craniofacial Anomalies. $1^{\text {st }}$ ed. Geneva: WHO; 2002. 